\title{
The Sanitation of Animal Waste Using Anaerobic Stabilization
}

\author{
Ingrid Papajová and Peter Juriš \\ Institute of Parasitology of the \\ Slovak Academy of Sciences \\ Slovak Republic
}

\section{Introduction}

Animal production pose potential hazards of environmental contamination with pathogenic microorganisms. These are particularly related to a subsequent storage processing and utilization of animal organic wastes (manure, fertilizer, wastewater, sludges etc.). A major source of pathogenic microorganisms in the environment are excrements from clinically and subclinically infected farm animals.

Handling, storage, treatment and use of different forms of animals excrements entails two principle problems: epizootological or epidemiological and hygienical. Solid excrements contain high numbers of common intestinal microflora (E. coli, faecal streptococci, lactobacilli etc.), bacteria that are pathogenic also for man (salmonellae, mycobacteria, listeriae etc.), protozoa (Isospora spp., Balantidium coli) and eggs or larvae of enteronematodes (Ascaris suum, Oesophagostomum sp., Trichuris suis etc.) (Lauková et al., 2000; Krupicer et al., 2000).

The parasitic propagative stages, mainly endoparasitic protozoa and helminths develop mostly outside their host's organism. A. suum eggs are hygienically the most problematic ones. They are amongst the helminth eggs most resistant to environmental factors and may survive in the nature for many years, therefore, they tend to accumulate in the environment (soil, water) and serve as an infectious entity for both man and animals (Papajová and Juriš, 2009). The cell wall of A suum egg is enveloped with an outer layer formed by acid polysaccharides and proteins, central layer consisting of proteins $(25 \%)$ and lipids $(75 \%$, particularly alpha glycosides). Thus this resistant cell wall protects eggs against effects of chemicals and drying (Eckert, 1992).

Regarding the spread of helminthoses, domestic animals (dogs, cats) are also of great importance because they live in a close contact with man. Infection and way of transmission of the disease depends on the way of breeding and on the breeding environment where the animal occurs. An important factor of the risk of infection transmission is also possibility of animal to move outside its housing (yard, move in nature), or the use of a dog (hunting or social). The most frequent way of transmission of parasitic diseases is through the contact (free-living animals with a domestic ones), or through contaminated environment with 
developmental stages (oocysts, eggs, larvae). Through faeces of infected dogs and cats the germs of parasitozoonoses spread into the environment. It is especially the case of cysts of intestinal parasitic protozoa - Entamoeba histolytica, Giardia intestinalis, Balantidium coli, Toxoplasma gondii, the eggs of tapeworms Dipylidium sp., Echinococcus sp. and parasitic nematodes (Antolová et al., 2004; Matsuo and Nakashio, 2005; Miterpáková et al., 2006). Regarding public health helminthozoonoses caused by Toxocara sp. (in dogs) and Ascaris sp. (in pigs) are very significant, especially due to their zoonotic character connected with the syndrome larva migrans.

Humans became infected usually orally (per os) by ingestion of substrates (soil, vegetables, etc.) with embryonated Toxocara eggs. Many symptoms are associated with this infection, including changes in blood cell counts and affection of various organs, as the ascaris larvae can migrate throughout the body. The symptoms of infection are often non-specific and may be mistaken with those of other infectious agents (common viral diseases, diarrhoea) or we may not observe any clinical signs. Toxocariasis manifests itself in two distinct forms: visceral, larva migrans visceralis, and ocular, larva migrans ocularis (Despommier, 2003).

A. suum infects pigs and is of major economical significance due to production losses linked to reduced feed conversion efficiency and losses to the mean industry associated with the condemnation of "milk-spot" livers (Dubinský et al., 2000). Ascaris infects over a quarter of the world's human population (1.47 billion people worldwide) and clinically affects $~ 335$ million people (Crompton, 1999).

The above-mentioned helminthozoonoses are classified among epidemiologically "low-risk" parasitozoonoses, because the propagative stages develop in the outdoor environment into the infectious stage and potentially secondarily contaminate the food chain. Therefore, a direct contact with infected animal, but also contaminated environment, or contaminated food chain (water, vegetables) are considered as a potential risk factor.

Attention has receantly been paid also to the problem of hygienical hazards in terms of the treatment and use of animal excrements and their application to soil as valuable nutrients for cultivated plants. The hazards are mainly connected with the quantity of continually produced solid and liquid wastes. The results is ecological disbalance, mainly with respect to environmental load with pathogenic microorgamism and nitrous organic substances. Animal organic wastes are also sources of greate amounts of gases releases. The most dangerous of them are ammonia and methane. Ammonia released into the atmosphere is irritating and toxic to the biotic component of the environment. On the other hand, animal excrements can supply essential plant nutrients and improve the fertility of soil by adding organic matter.

Therefore, to prevent health risks (for human as well as for animals) and odour nuisance from animal wastes, different methods for a satisfactory utilisation and sanitation have been researched (Schwartzbrod et al., 1989, Tofant et al., 1999; Juriš et al., 2000; Sasáková et al., 2005; Papajová and Juriš, 2009). There are big variations in the treatment of animal wastes (aerobic and anaerobic stabilization, composting etc.).

For the above-mentioned reasons our studies concentrated on anaerobic stabilization of liquid (slurry) and solid (manure, excrements) animal waste. The aims of our study were to 
monitor the physical-chemical changes in pig slurry treated by ecologically acceptable and energetically beneficial anaerobic stabilisation, changes in the properties of anaerobically digested slurry stored in ground lagoon and the effect of anaerobically digested slurry stores in ground lagoon on the survival of parasitic germs. The impact of lime (two types) on the survival of parasitic organisms in anaerobic stored manure and dog excrements mixed with hay, was also studied.

\section{Materials and methods}

\subsection{Parasitological methods}

To determine helminth eggs count in slurry (input and output samples from bioreactor and in lagoon samples - supernatant and sediment), $50 \mathrm{ml}$ from each of the 11 sample was taken and examined by a sedimentation-flotation mathod (Cherepanov, 1982).

A. suum eggs were isolated by dissection of a distal uterine part of female pig ascaris. The distal uterine ends were then removed to a glass homogenizer and processed. The water suspension of eggs was stored in an Erlenmayer flask in a refrigerator at $4^{\circ} \mathrm{C}$.

We used the "artificial contamination of lagoon and organic wastes" approach to make sure that there is s sufficient number of positive samples in our observations.

Model eggs were inoculated by a micropipette into polyurethane carriers, prepared according to Plachý and Juriš (1995), at a dose of 1000 eggs per one carrier. A porous cellular plastic - soft expanded polyurethane, commercially known as a plastic foam, was used as a material for the carriers. It is an additive product of polyisocyanates and compounds with a high content of hydroxylic groups. It consists of a network of interconnected cells, resembling a honeycomb. Its polyurethane structure allows for a sufficient contact of helminth eggs with the environment, preventing them from release and consequently improving their recovery (Picture 1). For mechanical protection the carriers were placed to perforated plastic net (Picture 2) before introducing them into the organic wastes. Three samples were taken and analysed at each sampling interval. The eggs were re-isolated from the inoculated carriers as follows: the carriers were cut into small pieces and washed in a mortar with $3 \cdot 5 \mathrm{ml}$ portions of saline, thoroughly stirred and filtered through a sieve into test tubes. After centrifugation, sediments were transferred to Petri dishes.

The viability of exposed unembryonated A. suum eggs was determined by incubation up to the embryonated stage (Picture 6) in a thermostat at $26^{\circ} \mathrm{C}$ for 21 days. Petri dishes with $A$. suum eggs were aerated daily with micropipette. The developmental ability of A. suum eggs was compared with that of the control eggs which were kept in distilled water under aerobic conditions.

\subsection{Physical and chemical methods}

The following changes in physical and chemical properties of the solid and liquid wastes were monitored: $\mathrm{pH}$, dry mater (DM), inorganic (IM) and organic (OM) matter, ammonium ions $\left(\mathrm{NH}_{4}{ }^{+}\right)$, total nitrogen $\left(\mathrm{N}_{\mathrm{t}}\right)$, chemical oxygen demand $(\mathrm{COD})$, soluble and insoluble substances and $\mathrm{C}: \mathrm{N}$ ratio. 


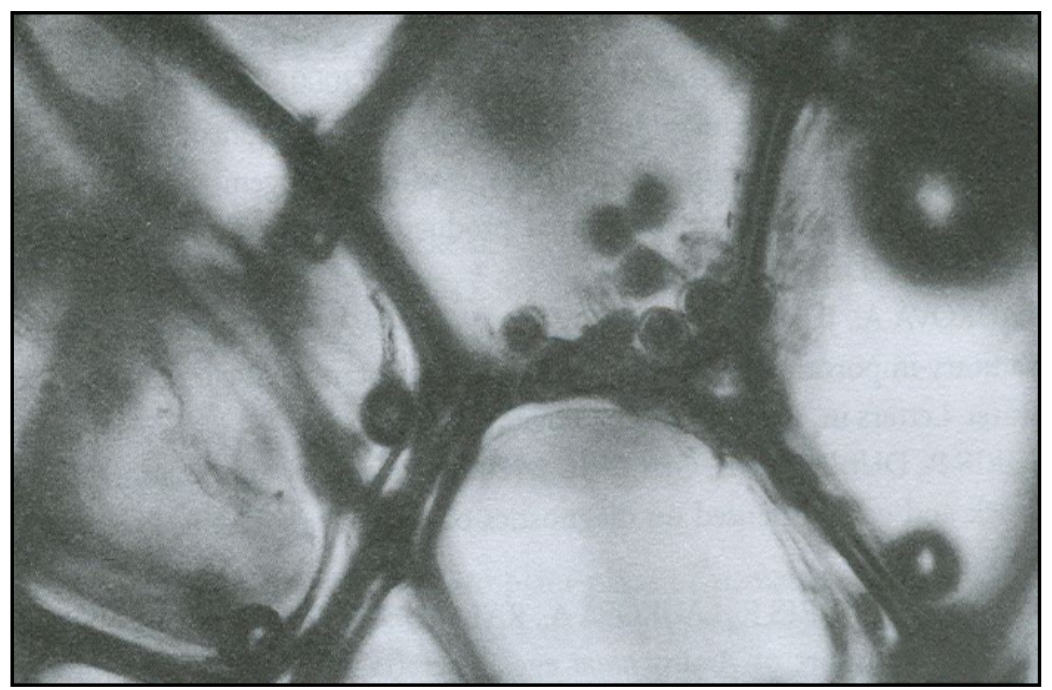

Picture 1. Polyurethane carrier with adhered A. suum eggs

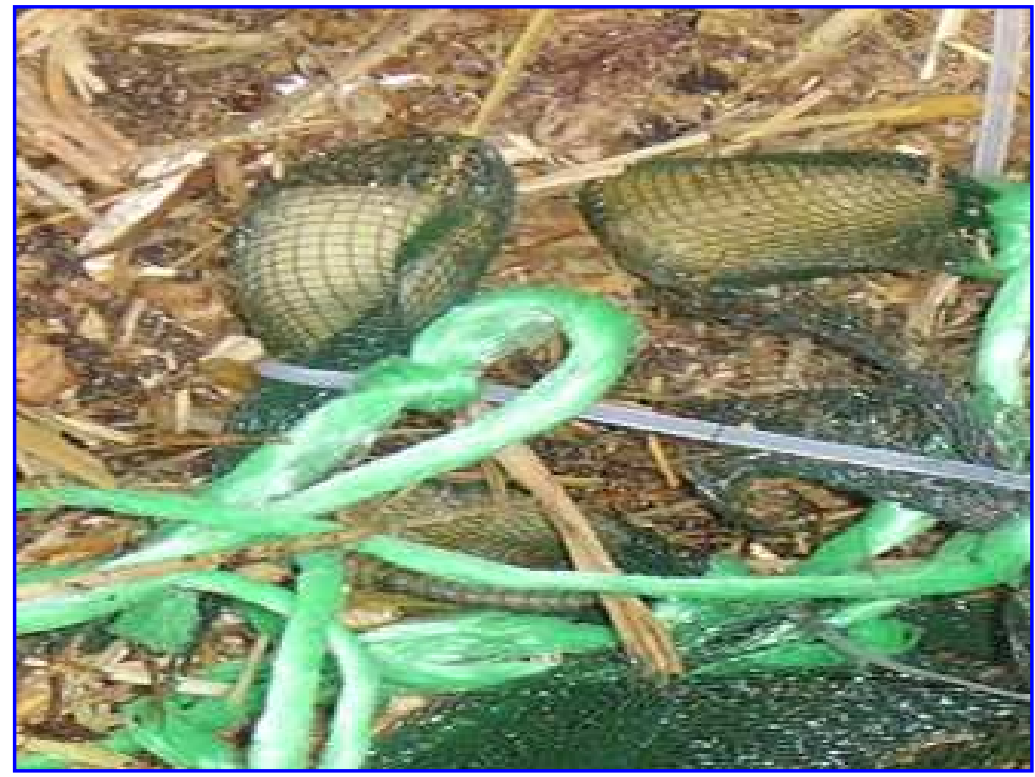

Picture 2. Perforated plastic nets with the carriers

The samples were examined for the $\mathrm{pH}(1: 10$ water extract) using a $\mathrm{pH}$ electrode (HACH Company, Loveland, Colorado, USA). Dry matter (drying at $105^{\circ} \mathrm{C}$ to a constant weight), residum-on-ignition $\left(550^{\circ} \mathrm{C}\right.$ for $\left.4 \mathrm{~h}\right)$, and water soluble ammonium nitrogen $\left(\mathrm{NH}_{4}{ }^{+}\right)$by titration (Mulvaney, 1996). Soluble and insoluble substances were determined by evaporation of the known amount of homogeneous sample filtrate on a water bath after 
absorption of insoluble substances on a filter, drying the evaporation residue at $105^{\circ} \mathrm{C}$ and determining its weight. COD was determined on the basis of organic substances oxidation in sample by potassium dichromate in sulfuric acid medium during 2-hour boiling in a COD reactor (HACH Company, Loveland, Colorado, USA). Portion of samples for $\mathrm{N}_{t}$ determinations were digested using a HACH-Digesdahl apparatus (HACH Company, Loveland, Colorado, USA). $\mathrm{N}_{t}$ was distilled with $\mathrm{NaOH}(40 \%)$ (Bremner, 1996). The $\mathrm{C}$ content was calculated according to the content of OM by the method of Navarro et al. (1993) to obtain the C:N ratio.

\subsection{Statistical analysis}

The physical and chemical properties $\left(\mathrm{pH}, \mathrm{DM}, \mathrm{IM}, \mathrm{OM}, \mathrm{NH}_{4}{ }^{+}, \mathrm{N}_{\mathrm{t}}\right.$ ) of solid animal wastes, as well as the number of demaged eggs were expressed as mean values \pm standard deviation $(\overline{\mathrm{X}} \pm \mathrm{SD})$.

Significance of differences between experimental and control groups of parasites were determined using Student t-test, ANOVA and Dunnet Multiple Comparison test at the levels of significance $0.05 ; 0.01$ and 0.001 (Statistica 6.0).

\section{Results}

\section{a) Anaerobic stabilisation of liquid animal wastes}

Investigations were carried out under operating conditions of the large-capacity pig farm in Slovak Republic (Picture 3). Technological equipment for anaerobic treatment of pig slurry on the principle of methanogenesis with the production of biogas was built up on the farm (Picture 4). Pig slurry was treated in the bioreactor $\left(2500 \mathrm{~m}^{3}\right)$ manufactured by Mostáren Brezno under the agreement with the firm BAUER Voitsberg. The stirring of the substrate in this reactor was done at the expense of energy of the generated biogas. Mean daily input of raw pig slurry in bioreactor of biogas plant varied between 78 and $144 \mathrm{~m}^{3}$. The volume of digested slurry after methanogenesis was equal to that of the input. Two lagoons were the part of the biogas plant. The volume of larger lagoon is $20000 \mathrm{~m}^{3}$ (Picture 5) and that of smaller lagoon is $5000 \mathrm{~m}^{3}$. Both lagoons serve as reservoirs of digested slurry. Liquid fraction from the smaller lagoon was carried away and spread on fields. The presence and survival of parasite eggs were studied in the larger lagoon. Samples were taken from raw slurry collecting basin before the inlet in to bioreactor (input samples), from outlet of digested slurry after methanogenesis in bioreactor (output samples), from supernatant (liquid fraction) and from lagoon sludge (solid fraction - sediment). The slurry samples for parasitological and physical and chemical examination were collected monthly during 29 month.

Slurry from the pig farm stored in the collecting basin showed a considerable variability during the period of study (Table 1). Compared with mean $\mathrm{pH}$ value of $7.12 \pm 0.26, \mathrm{pH}$ raw slurry in the month 11, 17 and 21 was lower, ranging between 6.61 and 6.95. The most conspicuous differences were recorded in DM content, which is most likely associated with the amount of process water use. The DM content in raw slurry determined during the period studied ranged from $0.81 \%$ to $5.30 \%$. The amount of $\mathrm{NH}_{4}{ }^{+}$in raw slurry was between $821 \mathrm{mg} \cdot \mathrm{l}^{-1}$ and $1774 \mathrm{mg} . \mathrm{l}^{-1}$. Chemical oxygen demand (COD) for that period varied from $2000 \mathrm{mg} . \mathrm{l}^{-1}$ to $22530 \mathrm{mg} . \mathrm{l}^{-1}$. The mean contents of $\mathrm{N}_{\mathrm{t}}$, in slurry was $1445 \pm 420 \mathrm{mg} . \mathrm{l}^{-1}$. 


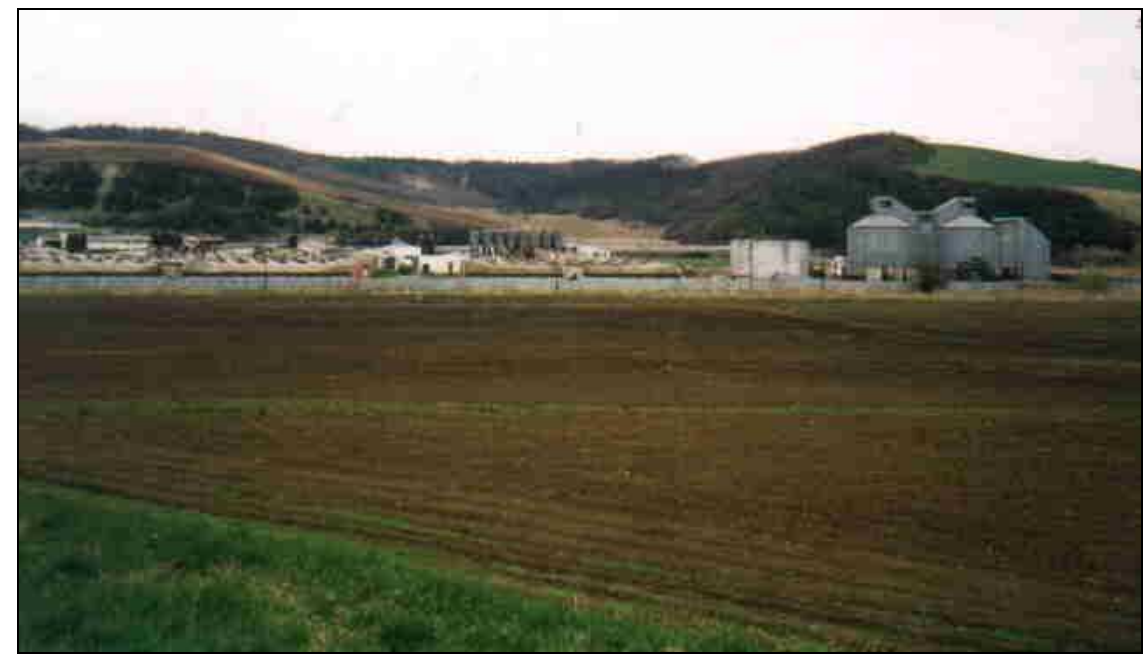

Picture 3. Large-capacity pig farm

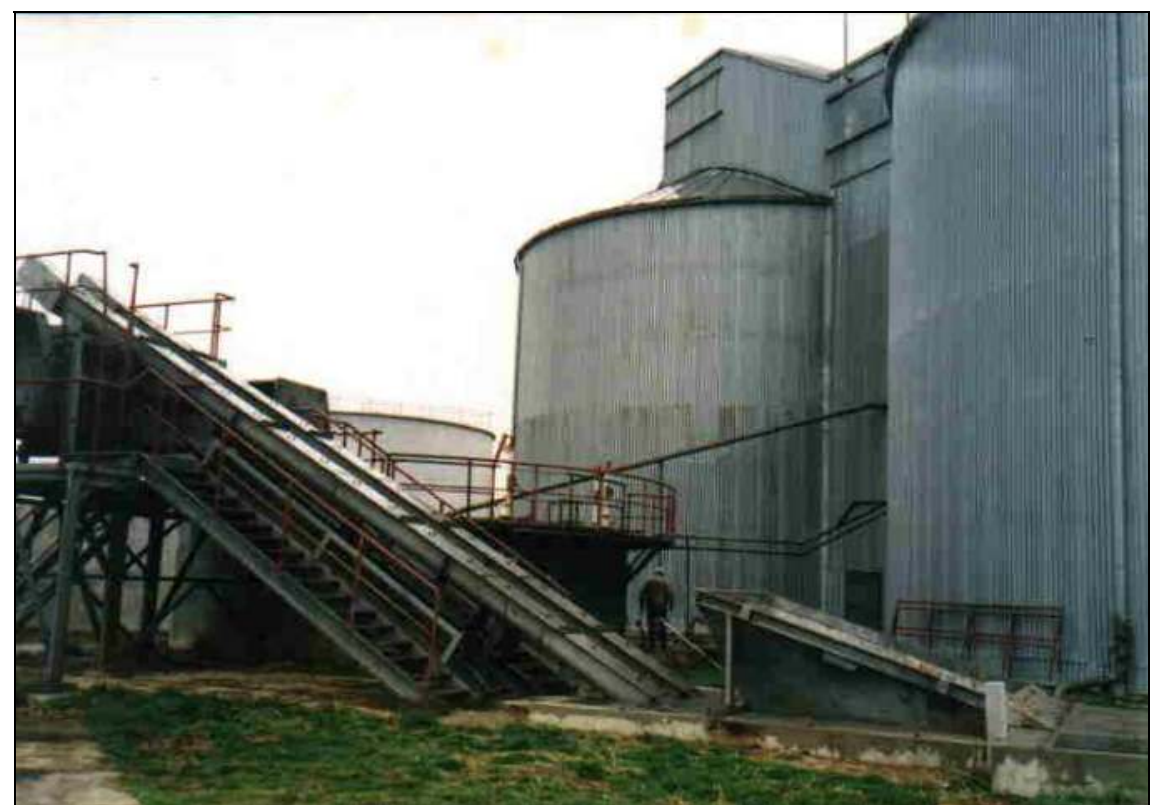

Picture 4. Bioreactors of biogas plant 


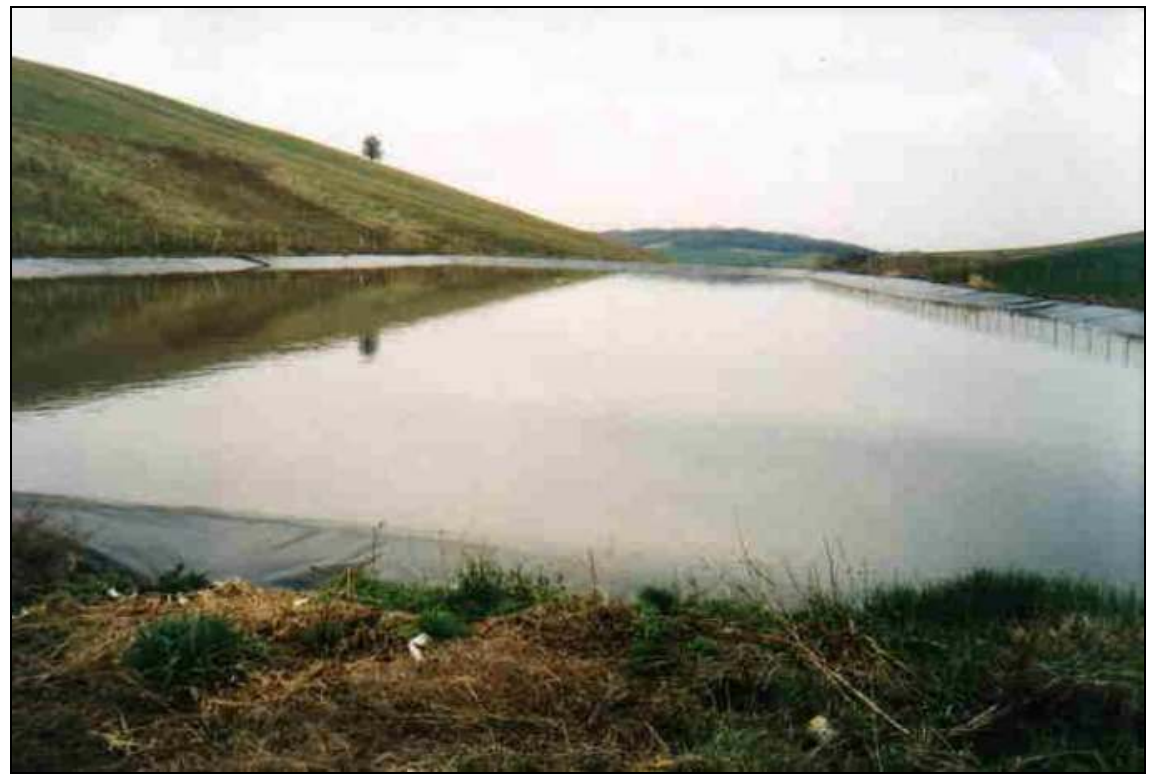

Picture 5. Large lagoon for storing digested pig slurry

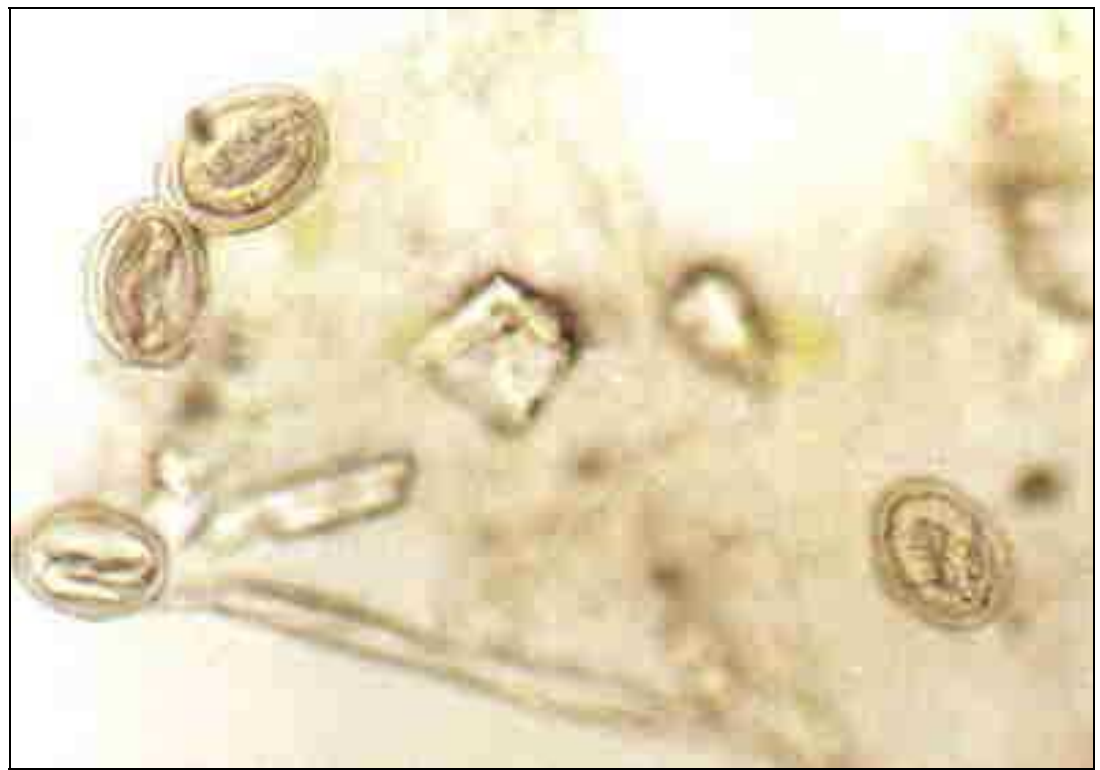

Picture 6. Embryonated A. suum eggs

Like raw pig slurry also slurry stabilised by anaerobic process showed variability of its physical-chemical parameters on its out flow from bioreactor (Table 2). Conspicuous differences were observed mainly in the dry mater content of anaerobically stabilized slurry. 
This is caused by the projected input, reckoning on the $5 \%$ of dry matter in raw pig slurry, but the mean dry matter content in raw slurry supplied to bioreactor was $1.96 \%$ and therefor poultry excrements had to be regularly added (average DM content $22.27 \%$ ) to pig slurry prior to its supply into bioreactor. Stabilized slurry outlet of bioreactor contained as much as $3.23 \pm 2.54 \% \mathrm{DM}$ on the average. Anaerobic digestion increased slurry $\mathrm{pH}$ which was ranging from 7.37 to 8.50. Compared with untreated slurry, anaerobic stabilization increased the content $\mathrm{NH}_{4}{ }^{+}$to $7.80 \pm 0.29 \mathrm{mg} . \mathrm{l}^{-1}$ on average. Concentration of $\mathrm{N}_{\mathrm{t}}$ was increased twice.

\begin{tabular}{|c|c|c|c|c|c|c|c|c|c|}
\hline $\begin{array}{l}\text { Storage } \\
\text { (month) }\end{array}$ & $\mathrm{pH}$ & $\begin{array}{l}\text { COD } \\
\left(\mathrm{mg}^{-l^{-1}}\right)\end{array}$ & $\begin{array}{l}\mathrm{DM} \\
(\%)\end{array}$ & $\begin{array}{l}\mathrm{IM} \\
(\%)\end{array}$ & $\begin{array}{l}\text { OM } \\
(\%)\end{array}$ & $\begin{array}{c}\text { Soluble } \\
\text { substances } \\
\left(\mathrm{mg} . \mathrm{l}^{-1}\right)\end{array}$ & $\begin{array}{c}\text { Insoluble } \\
\text { substances } \\
\left(\mathrm{mg} \cdot \mathbf{l}^{-1}\right)\end{array}$ & $\begin{array}{c}\mathrm{NH}_{4}{ }^{+} \\
\left.\text {(mg.l }{ }^{-1}\right)\end{array}$ & $\begin{array}{c}\mathrm{N}_{\mathrm{t}} \\
\left(\mathrm{mg} \cdot \mathrm{l}^{-1}\right)\end{array}$ \\
\hline 0 & 7.44 & 14833 & 2.75 & 31.87 & 68.13 & 11263 & 16264 & 1774 & 2419 \\
\hline 1 & 7.34 & 2000 & 0.84 & 51.42 & 48.58 & 5836 & 2612 & 1186 & 1401 \\
\hline 2 & 7.17 & 9297 & 0.95 & 43.04 & 56.96 & 4561 & 4897 & 821 & 1195 \\
\hline 3 & 7.03 & 13500 & 1.14 & 57.71 & 42.29 & 7757 & 3641 & 1202 & 1485 \\
\hline 4 & 7.00 & 20900 & 1.57 & 38.74 & 61.26 & 11095 & 4572 & 1078 & 1363 \\
\hline 5 & 7.35 & 14824 & 0.81 & 45.71 & 54.29 & 4895 & 3178 & 1037 & 1191 \\
\hline 6 & 7.36 & 13333 & 2.52 & 17.36 & 52.64 & 5366 & 19844 & 1247 & 1429 \\
\hline 11 & 6.61 & 21795 & 5.30 & 33.02 & 66.98 & - & - & 1695 & 1089 \\
\hline 17 & 6.95 & 12750 & 0.95 & 30.53 & 69.47 & 1000 & 8500 & 1478 & 1010 \\
\hline 21 & 6.95 & 22530 & 2.80 & 19.97 & 80.03 & 5870 & 22130 & 1358 & 1872 \\
\hline
\end{tabular}

Table 1. Physico-chemical properties of raw pig slurry (input sample of bioreactor) (COD chemical oxygen demand, DM - dry matter, IM - inorganic mater, OM - organic matter; $\mathrm{NH}_{4}{ }^{+}$- ammonium ions, $\mathrm{N}_{\mathrm{t}}$ - total nitrogen, - - not examined)

\begin{tabular}{|c|c|c|c|c|c|c|c|c|c|}
\hline $\begin{array}{l}\text { Storage } \\
\text { (month) }\end{array}$ & $\mathrm{pH}$ & $\begin{array}{l}\text { COD } \\
\left(\mathrm{mg}^{1-1}\right)\end{array}$ & $\begin{array}{l}\text { DM } \\
(\%)\end{array}$ & $\begin{array}{l}\text { IM } \\
(\%)\end{array}$ & $\begin{array}{l}\text { OM } \\
(\%)\end{array}$ & $\begin{array}{c}\text { Soluble } \\
\text { substances } \\
\left(\mathrm{mg} \cdot \mathrm{l}^{-1}\right)\end{array}$ & $\begin{array}{c}\text { Insoluble } \\
\text { substances } \\
\left(\text { mg.l-1) }^{-1}\right)\end{array}$ & $\begin{array}{c}\mathrm{NH}_{4}{ }^{+} \\
\left(\mathrm{mg} . \mathrm{l}^{-1}\right)\end{array}$ & $\begin{array}{c}\mathrm{N}_{\mathrm{t}} \\
\left(\mathrm{mg} \cdot \mathrm{l}^{-1}\right)\end{array}$ \\
\hline 0 & 8.50 & 36333 & - & - & - & - & - & 2633 & 6320 \\
\hline 1 & 7.74 & 10500 & 0.81 & 56.54 & 43.46 & 4739 & 3401 & 2204 & 2605 \\
\hline 2 & 7.63 & 17820 & 1.24 & 48.50 & 51.50 & 6134 & 6226 & 2157 & 2699 \\
\hline 3 & 7.80 & 8500 & 1.96 & 59.69 & 40.31 & 6192 & 13456 & 2045 & 2549 \\
\hline 4 & 7.69 & 17100 & 3.16 & 41.81 & 58.19 & 5965 & 5658 & 1933 & 3138 \\
\hline 5 & 7.77 & 6092 & 4.48 & 42.06 & 57.94 & 3225 & 41603 & 1898 & 1982 \\
\hline 6 & 7.92 & 2186 & 2.91 & 42.87 & 57.13 & 3555 & 25518 & 2437 & 3516 \\
\hline 11 & 7.88 & 4872 & 0.50 & 70.00 & 30.00 & - & - & 2171 & 1530 \\
\hline 17 & 7.37 & 7750 & 6.45 & 39.84 & 60.16 & 1389 & 63111 & 2248 & 1936 \\
\hline 21 & 7.66 & 42169 & 7.85 & 33.81 & 66.19 & 1333 & 77167 & 2655 & 3399 \\
\hline
\end{tabular}

Table 2. Physico-chemical properties of digested pig slurry (output sample of bioreactor) (COD - chemical oxygen demand, DM - dry matter, IM - inorganic mater, OM - organic matter; $\mathrm{NH}_{4}{ }^{+}$- ammonium ions, $\mathrm{N}_{\mathrm{t}}$ - total nitrogen, - - not examined) 
Anaerobically stabilized slurry was pumped from bioreactors into slurry ground lagoon for further storage. A long-term storage of digested slurry in lagoon is the most effective way of treatment resulting in a elimination of helminth eggs (Schwartzbrod et al., 1989). At the same time there is an increase in biogenic elements, especially of nitrogen and phophorus which are transformed into the forms acceptable by plants. Results of the chemical analysis of liquid fraction (supernatant) are presented in Table 3 and those of solid fraction (sludge) of lagoon in Table $4 . \mathrm{pH}$ of supernatant has not changed much over the period studied. Mean $\mathrm{pH}$ was $8.20 \pm 0.11 \%$. Sediment $\mathrm{pH}$ decreased during the first period of the study (month 0-6) and than again increased. Ammonia content was about equal in both the fraction. The highest content of $\mathrm{NH}_{4}{ }^{+}$was detected in spring month with its decrease observed in the course of study. $\mathrm{N}_{\mathrm{t}}$ contained by supernatant samples varied between 882 mg.l-1 to 2283 mg.l-1 (Table 3) and in sediment between $3571 \mathrm{mg} . \mathrm{l}^{-1}$ to $57831 \mathrm{mg} . \mathrm{l}^{-1}$ (Table 4). Sediment contained more DM and $\mathrm{N}_{\mathrm{t}}$ than supernatant (Tables 3,4 ).

\begin{tabular}{|c|c|c|c|c|c|c|c|c|c|}
\hline $\begin{array}{l}\text { Storage } \\
\text { (month) }\end{array}$ & $\mathrm{pH}$ & $\begin{array}{c}\text { COD } \\
\left(\mathrm{mg}^{-1} \mathbf{1}^{-1}\right)\end{array}$ & $\begin{array}{l}\text { DM } \\
(\%)\end{array}$ & $\begin{array}{l}\text { IM } \\
(\%)\end{array}$ & $\begin{array}{l}\text { OM } \\
(\%)\end{array}$ & $\begin{array}{c}\text { Soluble } \\
\text { substances } \\
\left(\mathrm{mg} \cdot \mathbf{l}^{-1}\right)\end{array}$ & $\begin{array}{c}\text { Insoluble } \\
\text { substances } \\
\left(\mathrm{mg} . \mathrm{l}^{-1}\right)\end{array}$ & $\begin{array}{c}\mathrm{NH}_{4}{ }^{+} \\
\left.\text {(mg. } 1^{-1}\right)\end{array}$ & $\begin{array}{c}\mathrm{N}_{\mathrm{t}} \\
\left.\text { (mg. } \mathrm{l}^{-1}\right)\end{array}$ \\
\hline 0 & 8.30 & 4500 & 0.50 & 61.06 & 38.94 & 4416 & 581 & 1737 & 1910 \\
\hline 1 & 8.20 & 4000 & 0.50 & 70.39 & 29.61 & 4808 & 174 & 1307 & 1428 \\
\hline 2 & 8.17 & 2002 & 0.68 & 57.58 & 42.42 & 6579 & 239 & 1345 & 1569 \\
\hline 3 & 8.34 & 3500 & 0.66 & 57.75 & 42.25 & 5340 & 1272 & 1111 & 1214 \\
\hline 4 & 8.10 & 7600 & 0.93 & 56.04 & 43.96 & 6085 & 3177 & 1408 & 1662 \\
\hline 5 & 8.08 & 6552 & 0.87 & 52.76 & 47.24 & 3600 & 5255 & 1135 & 1172 \\
\hline 6 & 8.29 & 1530 & 0.71 & 57.59 & 42.41 & 2748 & 4337 & 1107 & 1223 \\
\hline 13 & 8.21 & 7059 & 0.70 & 55.53 & 44.47 & 5954 & 1083 & 1863 & 2283 \\
\hline 14 & 8.07 & 818 & 1.68 & 46.60 & 53.40 & 5588 & 11217 & 1569 & 1569 \\
\hline 15 & 8.28 & 1904 & 0.66 & 54.83 & 45.17 & 5325 & 1284 & 1331 & 1317 \\
\hline 16 & 8.21 & 5385 & 0.63 & 56.38 & 43.62 & 4483 & 1806 & 896 & 882 \\
\hline 17 & 8.29 & 8605 & 0.60 & 54.27 & 45.73 & 3501 & 2524 & 616 & 1415 \\
\hline 23 & 8.32 & 3333 & 0.35 & 71.43 & 28.57 & 2128 & 1372 & 672 & 1016 \\
\hline 29 & 7.95 & 5000 & 0.75 & 45.33 & 54.67 & 3000 & 4500 & 862 & 1031 \\
\hline
\end{tabular}

Table 3. Physico-chemical properties of supernatant from stabilized pig slurry stored in lagoon (COD - chemical oxygen demand, DM - dry matter, IM - inorganic mater, OM - organic matter; $\mathrm{NH}_{4}{ }^{+}$- ammonium ions, $\mathrm{N}_{\mathrm{t}}$ - total nitrogen, - - not examined)

A. sum eggs and Oesophagostomum sp. eggs were rarely detected in slurry on the input and also on the output of bioreactor (Table 5). Similar results of helminths eggs occurrence in anaerobic slurry treatment were also presented by Juriš et al. (1996), No helminth eggs were found in the supernatant of digested slurry from the lagoon. A. suum eggs were found in sediment (Table 5).

High percentage of devitalised unembryonated A. suum eggs $(47.46 \pm 0.78 \%)$ stored 11 months (from May - month 13 to March - month 23) in a ground slurry lagoon points to the impact of high concentration of $\mathrm{NH}_{4}{ }^{+}$(max. $5358 \mathrm{mg} . \mathrm{l}^{-1}$ in sediment compared to $1863 \mathrm{mg} . \mathrm{l}^{-}$ 1 in supernatant), which are releasing during a period of time from an open area of the 
ground lagoon, and nitrogen (max. 9854 mg.l-1 in sediment compared to 2283 mg.l-1 in supernatant) on devitalization of developmental stages of endoparasites. The number of devitalised A. suum eggs increased towards to the bottom of lagoon. In the control groups, only $19.60 \pm 1.80 \%$ of $A$. suum eggs were devitalized (Table 6 ).

\begin{tabular}{|c|c|c|c|c|c|c|c|c|c|}
\hline $\begin{array}{l}\text { Storage } \\
\text { (month) }\end{array}$ & $\mathrm{pH}$ & $\begin{array}{l}\text { COD } \\
\left(\mathrm{mg}^{1-1}\right)\end{array}$ & $\begin{array}{l}\text { DM } \\
(\%)\end{array}$ & $\begin{array}{l}\text { IM } \\
(\%)\end{array}$ & $\begin{array}{l}\text { OM } \\
(\%)\end{array}$ & $\begin{array}{c}\text { Soluble } \\
\text { substances } \\
\left(\mathrm{mg}^{1-1}\right)\end{array}$ & $\begin{array}{c}\text { Insoluble } \\
\text { substances } \\
\left(\mathrm{mg} . \mathrm{l}^{-1}\right)\end{array}$ & $\begin{array}{l}\mathrm{NH}_{4}^{+} \\
\text {(mg.l-1) }\end{array}$ & $\begin{array}{c}\mathrm{N}_{\mathrm{t}} \\
\left(\mathrm{mg} . \mathrm{l}^{-1}\right)\end{array}$ \\
\hline 0 & 8.37 & 9333 & 1.17 & 49.21 & 50.79 & 1885 & 2138 & 5778 & 5963 \\
\hline 1 & 8.13 & 11000 & 1.17 & 48.26 & 51.74 & 1681 & 1830 & 5635 & 6041 \\
\hline 2 & 8.07 & 6170 & 1,70 & 43.21 & 56.79 & 1643 & 2241 & 7344 & 9652 \\
\hline 3 & 8.09 & 4500 & 1.28 & 31.52 & 52.88 & 1363 & 1625 & 4042 & 6782 \\
\hline 4 & 7.90 & 55100 & 1.12 & 34.90 & 65.10 & 1359 & 2437 & 3913 & 7298 \\
\hline 5 & 8.08 & 8965 & - & - & - & 1149 & 4755 & - & - \\
\hline 6 & 7.87 & 7322 & - & - & - & 1541 & - & - & - \\
\hline 13 & - & - & - & - & - & - & -- & - & - \\
\hline 14 & 7.73 & 6367 & - & - & - & 5358 & 9854 & - & - \\
\hline 15 & 8.24 & 2494 & 1.72 & 32.11 & 67.89 & 840 & 1233 & 5513 & 11658 \\
\hline 16 & 8.17 & 5897 & 0.73 & 51.16 & 48.84 & 1989 & 938 & 3740 & 3571 \\
\hline 17 & 8.12 & 27186 & 1.19 & 45.41 & 54.59 & 915 & 1387 & 5402 & 6493 \\
\hline 23 & 8.11 & - & 13.01 & 55.56 & 44.44 & 308 & 3909 & 72289 & 57831 \\
\hline 29 & - & - & - & - & - & - & - & - & - \\
\hline
\end{tabular}

Table 4. Physico-chemical properties of sediment from stabilized pig sllury stored in lagoon (COD - chemical oxygen demand, DM - dry matter, IM - inorganic mater, OM - organic matter; $\mathrm{NH}_{4}{ }^{+}$- ammonium ions, $\mathrm{N}_{\mathrm{t}}$ - total nitrogen, - - not examined)

\begin{tabular}{|c|c|c|c|c|c|c|c|c|c|c|c|c|c|c|c|c|}
\hline \multirow{2}{*}{ Slurry } & \multicolumn{16}{|c|}{ Storage (month) and occurence of eggs per litre sample } \\
\hline & 0 & 1 & 2 & 3 & 4 & 5 & 6 & 11 & 13 & 14 & 15 & 16 & 17 & 21 & 23 & 29 \\
\hline $\begin{array}{l}\text { Input } \\
\text { (raw) }\end{array}$ & Oe-2 & ND & ND & A-5 & ND & ND & ND & - & - & - & - & - & - & ND & ND & ND \\
\hline $\begin{array}{l}\text { Output } \\
\text { (digested) }\end{array}$ & A-2 & ND & ND & ND & ND & ND & ND & - & - & - & - & - & - & ND & A-1 & ND \\
\hline $\begin{array}{l}\text { Supernatant } \\
\text { (lagoon) }\end{array}$ & ND & ND & ND & ND & ND & ND & ND & ND & ND & ND & ND & ND & ND & ND & ND & ND \\
\hline $\begin{array}{l}\text { Sediment } \\
\text { (lagoon) }\end{array}$ & ND & ND & A- 6 & ND & ND & ND & ND & ND & ND & ND & ND & ND & ND & A-2 & ND & ND \\
\hline
\end{tabular}

Table 5. Occurence of helminth eggs in slurry and in lagoon (A - A. suum eggs, Oe Oesophagostomum sp. eggs, ND - not detected, - - not examined) 


\begin{tabular}{ccc}
\hline & \multicolumn{2}{c}{ Damaged A. suum eggs $(\overline{\mathrm{X}} \% \pm \mathrm{SD})$} \\
\cline { 2 - 3 } Storage (month) & Lagoon & Control \\
\hline May (13) & $16.23 \pm 3.22$ & $14.80 \pm 2.43$ \\
June (14) & $38.27 \pm 2.51$ & $15.79 \pm 2.44$ \\
September (17) & $40.37 \pm 2.94$ & $18.23 \pm 1.22$ \\
March (23) & $47.46 \pm 0.78$ & $19.60 \pm 1.80$ \\
\hline
\end{tabular}

Table 6. Damage of A. suum eggs during long term storage of anaerobic stabilized pig slurry in lagoon

\section{b) Anaerobic stabilisation of solid animal wastes}

The effect of anaerobic stabilisation of solid animal wastes (manure, dog excrements) with or without addition of lime on the survival of parasitic germs were studied under laboratory conditions. Two types of lime was used in the experiment: 1. quality dust lime and 2. dust rejects from lime production caught on the electrostatic precipitator. General characteristics of tested lime are given in Table 7.

\begin{tabular}{ccc}
\hline & Quality dust lime & Dust rejects \\
\hline $\mathrm{CaO}+\mathrm{MgO}$ & $\min .95 .0 \%$ & $\min .82 .0 \%$ \\
$\mathrm{MgO}$ & $\max .5 .0 \%$ & $\max .3 .5 \%$ \\
$\mathrm{CO}_{2}$ & $\max .2 .5 \%$ & $\max .11 .0 \%$ \\
Granularity & $0-0.2 \mathrm{~mm}$ & $0-1.0 \mathrm{~mm}$ \\
\hline
\end{tabular}

Table 7. Physico-chemical properties of the tested types of lime

Pig manure (M) and dog excrements mixed with hay in the ratio of 1:5 (D) were used in the experiment. Organic wastes were mixed with tested lime in a different concentration and periodically stirred. The following variations were investigated in comparison to untreated (control) manure (CM) and untreated dog droppings (CD):

a. manure mixed with quality dust lime in a concentration of 20 g. $\mathrm{kg}^{-1}$ (ML20)

b. manure mixed with dust rejects in a concentration of $20 \mathrm{~g} \cdot \mathrm{kg}^{-1}$ (M20)

c. dog droppings mixed with dust rejects in a concentration of 20 g.kg-1 (D20),

d. dog droppings mixed with dust rejects in a concentration of 70 g.kg-1 (D70).

Samples for parasitological and physical and chemical examinations were collected after 0 , 1, 3, 8, 14, 36 (UM, ML20 and M20) and after 0, 1, 2, 3, 7, 8, 9, 10, 14, 73 (UD, D20, D70) days of exposure. Three samples were taken and analysed at each of the given sampling intervals.

The physical and chemical properties of treated manure and dog excrements are given in Tables 8 - 13. Comparison of the changes in The physical and chemical properties of organic material during anaerobic stabilisation with or withou dust rejects is given in Fig. $1-5$. 


\begin{tabular}{cccccccc}
\hline $\begin{array}{c}\text { Storage } \\
\text { (days) }\end{array}$ & $\mathbf{p H}$ & $\begin{array}{c}\mathbf{D M} \\
\mathbf{( \% )}\end{array}$ & $\begin{array}{c}\mathbf{I M} \\
\mathbf{( \% )}\end{array}$ & $\begin{array}{c}\mathbf{O M} \\
\mathbf{( \% )}\end{array}$ & $\begin{array}{c}\mathbf{N H}_{\mathbf{4}}{ }^{+} \\
\mathbf{( m g . k g - 1} \mathbf{D M})\end{array}$ & $\begin{array}{c}\mathbf{N}_{\mathbf{t}} \\
(\mathbf{m g} \cdot \mathbf{k g}-\mathbf{D} \mathbf{D})\end{array}$ & $\mathbf{C}: \mathbf{N}$ \\
\hline $\mathbf{0}$ & $8.47 \pm 0.58$ & $33.22 \pm 6.88$ & $8.09 \pm 2.57$ & $91.91 \pm 2.57$ & $120.89 \pm 7.05$ & $13789.52 \pm 2356.62$ & $34.27: 1$ \\
$\mathbf{1}$ & $8.57 \pm 0.02$ & $20.66 \pm 4,29$ & $10.41 \pm 0.72$ & $89.58 \pm 0.72$ & $257.65 \pm 10.10$ & $51930.16 \pm 421.47$ & $8.84: 1$ \\
$\mathbf{3}$ & $9.52 \pm 0.06$ & $28.06 \pm 5.41$ & $6.03 \pm 0.11$ & $93.37 \pm 0.11$ & $176.37 \pm 8.09$ & $46522.24 \pm 2310.56$ & $10.27: 1$ \\
$\mathbf{8}$ & $9.28 \pm 0.02$ & $23.50 \pm 4.12$ & $8.34 \pm 2.66$ & $91.66 \pm 2.66$ & $214.60 \pm 7.92$ & $49872.94 \pm 1715.15$ & $9.41: 1$ \\
$\mathbf{1 4}$ & $8.26 \pm 0.02$ & $14.99 \pm 0.39$ & $9.12 \pm 1.14$ & $90.88 \pm 1.14$ & $510.81 \pm 11.32$ & $58608.01 \pm 2701.82$ & $7.97: 1$ \\
$\mathbf{3 6}$ & $8.27 \pm 0.06$ & $14.36 \pm 0.12$ & $9.48 \pm 0.13$ & $90.52 \pm 0.13$ & $48.75 \pm 2.80$ & $32698.26 \pm 2378.98$ & $14.13: 1$ \\
\hline
\end{tabular}

Table 8. Physico-chemical properties of the pig manure during anaerobic stabilization (CM) (DM - dry matter, IM - inorganic mater, $\mathrm{OM}$ - organic matter; $\mathrm{NH}_{4}{ }^{+}$- ammonium ions, $\mathrm{N}_{\mathrm{t}}$ total nitrogen)

\begin{tabular}{cccccccc}
\hline $\begin{array}{c}\text { Storage } \\
\text { (days) }\end{array}$ & $\mathbf{p H}$ & $\begin{array}{c}\mathbf{D M} \\
\mathbf{( \% )}\end{array}$ & $\begin{array}{c}\mathbf{I M} \\
\mathbf{( \% )}\end{array}$ & $\begin{array}{c}\mathbf{O M} \\
\mathbf{( \% )}\end{array}$ & $\begin{array}{c}\mathbf{N H}_{\mathbf{4}}{ }^{+} \\
\left.\mathbf{m g}_{\mathbf{m g}} \mathbf{- 1} \mathbf{D M}\right)\end{array}$ & $\begin{array}{c}\mathbf{N}_{\mathbf{t}} \\
\left.\mathbf{( m g . k g}^{-1} \mathbf{D M}\right)\end{array}$ & $\mathbf{C : N}$ \\
\hline $\mathbf{0}$ & $8.47 \pm 0.58$ & $33.22 \pm 6.88$ & $8.09 \pm 2.57$ & $91.91 \pm 2.57$ & $120.89 \pm 7.05$ & $13789.52 \pm 2356,62$ & $34.27: 1$ \\
$\mathbf{1}$ & $12.97 \pm 0.02$ & $41.57 \pm 2.46$ & $58.88 \pm 17.14$ & $41.12 \pm 17.14$ & $69.64 \pm 4.28$ & $125901.56 \pm 873.31$ & $8.11: 1$ \\
$\mathbf{3}$ & $12.76 \pm 0.01$ & $45.96 \pm 3.72$ & $39.91 \pm 7.76$ & $60.09 \pm 7.76$ & $111.75 \pm 9.84$ & $18866,51 \pm 3349.86$ & $16.32: 1$ \\
$\mathbf{8}$ & $10.39 \pm 0.01$ & $26.91 \pm 2.16$ & $16.95 \pm 2.67$ & $83.05 \pm 2.67$ & $236.01 \pm 7.05$ & $52051.28 \pm 1482.36$ & $8.17: 1$ \\
$\mathbf{1 4}$ & $8.29 \pm 0.01$ & $21.47 \pm 5.22$ & $14.36 \pm 3.41$ & $85.64 \pm 3.41$ & $326.22 \pm 17.94$ & $56824.87 \pm 2746.13$ & $7.72: 1$ \\
$\mathbf{3 6}$ & $8.29 \pm 0.01$ & $20.11 \pm 2.32$ & $13.41 \pm 1.12$ & $86.19 \pm 1.12$ & $225.21 \pm 22.47$ & $71771.76 \pm 1722.51$ & $6.14: 1$ \\
\hline
\end{tabular}

Table 9. Physico-chemical properties of the pig manure mixed with dust rejects in a concentration of $20 \mathrm{~g} \cdot \mathrm{kg}^{-1}$ during anaerobic stabilization (M20) (DM - dry matter, IM inorganic mater, $\mathrm{OM}$ - organic matter; $\mathrm{NH}_{4}{ }^{+}$- ammonium ions, $\mathrm{N}_{\mathrm{t}}$ - total nitrogen)

\begin{tabular}{cccccccc}
\hline $\begin{array}{c}\text { Storage } \\
\text { (days) }\end{array}$ & $\mathbf{p H}$ & $\begin{array}{c}\mathbf{D M} \\
\mathbf{( \% )}\end{array}$ & $\begin{array}{c}\mathbf{I M} \\
\mathbf{( \% )}\end{array}$ & $\begin{array}{c}\mathbf{O M} \\
\mathbf{( \% )}\end{array}$ & $\begin{array}{c}\mathbf{N H}_{\mathbf{4}}{ }^{+} \\
\mathbf{( m g . k g - 1} \mathbf{D M})\end{array}$ & $\begin{array}{c}\mathbf{N}_{\mathbf{t}} \\
\mathbf{( m g . k g - 1} \mathbf{D M})\end{array}$ & $\mathbf{C}: \mathbf{N}$ \\
\hline $\mathbf{0}$ & $8.47 \pm 0.58$ & $33.22 \pm 6.88$ & $8.09 \pm 2.57$ & $91.91 \pm 2.57$ & $120.89 \pm 7.05$ & $13789.52 \pm 2356,62$ & $34.27: 1$ \\
$\mathbf{1}$ & $12.86 \pm 0.03$ & $30.33 \pm 3.87$ & $48.90 \pm 15.23$ & $51.10 \pm 15.23$ & $126.24 \pm 9.84$ & $37815.69 \pm 1860.53$ & $6.97: 1$ \\
$\mathbf{3}$ & $12.96 \pm 0.01$ & $37.31 \pm 3.89$ & $57.40 \pm 5.92$ & $42.60 \pm 5.92$ & $130.15 \pm 9.01$ & $35790.24 \pm 2332.63$ & $6.08: 1$ \\
$\mathbf{8}$ & $11.56 \pm 0.02$ & $25.37 \pm 0.95$ & $48.67 \pm 3.35$ & $51.33 \pm 3.35$ & $176.67 \pm 10.10$ & $81616.08 \pm 3704.40$ & $3.21: 1$ \\
$\mathbf{1 4}$ & $9.36 \pm 0.01$ & $20.30 \pm 2.17$ & $34.12 \pm 1.12$ & $65.88 \pm 1.12$ & $206.99 \pm 17.83$ & $44057.78 \pm 2515.94$ & $7.66: 1$ \\
$\mathbf{3 6}$ & $8.76 \pm 0.01$ & $20.08 \pm 1.56$ & $32.48 \pm 3.46$ & $67.52 \pm 3.46$ & $181.37 \pm 25.75$ & $65746.86 \pm 2677.51$ & $5.25: 1$ \\
\hline
\end{tabular}

Table 10. Physico-chemical properties of the pig manure mixed with quick lime in a concentration of 20 g.kg-1 during anaerobic stabilization (ML20) (DM - dry matter, IM inorganic mater, $\mathrm{OM}$ - organic matter; $\mathrm{NH}_{4}{ }^{+}$- ammonium ions, $\mathrm{N}_{\mathrm{t}}$ - total nitrogen, - - not examined) 


\begin{tabular}{cccccccc}
\hline $\begin{array}{c}\text { Storage } \\
\text { (days) }\end{array}$ & $\mathbf{p H}$ & $\begin{array}{c}\mathbf{D M} \\
\mathbf{( \% )}\end{array}$ & $\begin{array}{c}\mathbf{I M} \\
\mathbf{( \% )}\end{array}$ & $\begin{array}{c}\mathbf{O M} \\
\mathbf{( \% )}\end{array}$ & $\begin{array}{c}\mathbf{N H}_{\mathbf{4}}{ }^{+} \\
\left.\mathbf{( m g . k g}^{-1} \mathbf{D M}\right)\end{array}$ & $\begin{array}{c}\mathbf{N}_{\mathbf{t}} \\
\left.\mathbf{m g}_{\mathbf{m}} \mathbf{- 1} \mathbf{D M}\right)\end{array}$ & $\mathbf{C}: \mathbf{N}$ \\
\hline $\mathbf{0}$ & $9.08 \pm 0.01$ & $35.66 \pm 1.83$ & $11.33 \pm 0.01$ & $88.67 \pm 0.01$ & $219.07 \pm 55,70$ & $40758.43 \pm 1416.02$ & $11.15: 1$ \\
$\mathbf{1}$ & $8.57 \pm 0.01$ & $34.66 \pm 0.11$ & $14.29 \pm 1.77$ & $85.71 \pm 1.77$ & $232.05 \pm 23,57$ & $39116.17 \pm 207.87$ & $11.24: 1$ \\
$\mathbf{2}$ & $9.61 \pm 0.01$ & $35.20 \pm 4.23$ & $19.21 \pm 4.89$ & $80.79 \pm 4.89$ & $395,72 \pm 2,48$ & $41116.07 \pm 1205.26$ & $10.12: 1$ \\
$\mathbf{3}$ & $9.78 \pm 0.01$ & $37.56 \pm 1.93$ & $22.33 \pm 1.06$ & $77.67 \pm 1.06$ & $309.78 \pm 95.04$ & $44207.73 \pm 3222.05$ & $9.05: 1$ \\
$\mathbf{7}$ & $9.01 \pm 0.01$ & $37.17 \pm 0.29$ & $20.58 \pm 0.48$ & $79.42 \pm 0.48$ & $370,89 \pm 8.22$ & $23346.91 \pm 5147.86$ & $18.06: 1$ \\
$\mathbf{8}$ & $9.39 \pm 0.02$ & $33.21 \pm 0.18$ & $22.46 \pm 1.97$ & $77.54 \pm 1.97$ & $82.25 \pm 2.55$ & $12152.63 \pm 77.34$ & $32.74: 1$ \\
$\mathbf{9}$ & $9.55 \pm 0.02$ & $29.81 \pm 3.03$ & $31.17 \pm 3.54$ & $68.83 \pm 3.54$ & $132.58 \pm 72.91$ & $6556.92 \pm 818.39$ & $54.92: 1$ \\
$\mathbf{1 0}$ & $9.49 \pm 0.03$ & $31.96 \pm 1.13$ & $31.46 \pm 0.36$ & $68.54 \pm 0,36$ & $124.07 \pm 0.16$ & $6217.49 \pm 27.34$ & $57.31: 1$ \\
$\mathbf{1 4}$ & $9.34 \pm 0.03$ & $53.90 \pm 4.33$ & $32.32 \pm 0.91$ & $67.68 \pm 0.91$ & $138.03 \pm 7.41$ & $5435.17 \pm 2904.64$ & $76.40: 1$ \\
$\mathbf{7 3}$ & $8.51 \pm 0.03$ & $86.31 \pm 0.23$ & $17.99 \pm 1.73$ & $82.01 \pm 1.73$ & $28.09 \pm 3.26$ & $9159.80 \pm 1327.01$ & $46.33: 1$ \\
\hline
\end{tabular}

Table 11. Physico-chemical properties of the dog excrements during anaerobic stabilization (CD) (DM - dry matter, IM - inorganic mater, OM - organic matter; $\mathrm{NH}_{4}{ }^{+}$- ammonium ions, $\mathrm{N}_{\mathrm{t}}$ - total nitrogen, - - not examined)

\begin{tabular}{cccccccc}
\hline $\begin{array}{c}\text { Storage } \\
\text { (days) }\end{array}$ & $\mathbf{p H}$ & $\begin{array}{c}\mathbf{D M} \\
\mathbf{( \% )}\end{array}$ & $\begin{array}{c}\mathbf{I M} \\
\mathbf{( \% )}\end{array}$ & $\begin{array}{c}\mathbf{O M} \\
\mathbf{( \% )}\end{array}$ & $\begin{array}{c}\mathbf{N H}_{\mathbf{4}}{ }^{+} \\
\left.\mathbf{m g}_{\mathbf{m}} \mathbf{- 1} \mathbf{D M}\right)\end{array}$ & $\begin{array}{c}\mathbf{N}_{\mathbf{t}} \\
\mathbf{( m g . k g - 1} \mathbf{D M})\end{array}$ & $\mathbf{C : N}$ \\
\hline $\mathbf{0}$ & $8.41 \pm 0.05$ & $37.21 \pm 0.01$ & $16.04 \pm 4.81$ & $83.96 \pm 4.81$ & $400.57 \pm 47.84$ & $45177.35 \pm 4724.10$ & $9.64: 1$ \\
$\mathbf{1}$ & $11.21 \pm 0.02$ & $44.47 \pm 0.91$ & $39.37 \pm 0.10$ & $60.63 \pm 0.10$ & $12.51 \pm 8.65$ & $32262.40 \pm 4212.96$ & $9.66: 1$ \\
$\mathbf{2}$ & $9.34 \pm 0.03$ & $56.45 \pm 15.15$ & $62.63 \pm 19.97$ & $37.37 \pm 19.97$ & $36.17 \pm 7.84$ & $16218.95 \pm 2664.67$ & $12.44: 1$ \\
$\mathbf{3}$ & $8.58 \pm 0.01$ & $57.28 \pm 33.23$ & $60.29 \pm 28.06$ & $39.71 \pm 28.06$ & $645.10 \pm 362.56$ & $45266.01 \pm 19058.77$ & $4.23: 1$ \\
$\mathbf{7}$ & $9.08 \pm 0.01$ & $45.11 \pm 6.79$ & $43.58 \pm 0.64$ & $56.42 \pm 0.64$ & $225.45 \pm 91.01$ & $22104.48 \pm 11603.90$ & $15.37: 1$ \\
$\mathbf{8}$ & $9.13 \pm 0.01$ & $43.90 \pm 2.84$ & $41.87 \pm 0.96$ & $58.13 \pm 0.96$ & $439.78 \pm 141.28$ & $18254.61 \pm 1760.78$ & $16.74: 1$ \\
$\mathbf{9}$ & $9.27 \pm 0.05$ & $68.66 \pm 1.32$ & $27.61 \pm 0.60$ & $72.39 \pm 0.60$ & $398.59 \pm 2.51$ & $18083.74 \pm 303.53$ & $20.86: 1$ \\
$\mathbf{1 0}$ & $9.12 \pm 0.04$ & $64.15 \pm 0.16$ & $42.28 \pm 0.71$ & $57.64 \pm 0.71$ & $349.02 \pm 10.08$ & $16966.79 \pm 191.73$ & $17.70: 1$ \\
$\mathbf{1 4}$ & $8.91 \pm 0.03$ & $60.02 \pm 0.98$ & $49.20 \pm 4.49$ & $50.80 \pm 4.49$ & $338.50 \pm 24,95$ & $17963.19 \pm 457.92$ & $14.71: 1$ \\
$\mathbf{7 3}$ & $8.69 \pm 0.01$ & $89.06 \pm 0.01$ & $38.85 \pm 3.90$ & $61.15 \pm 3.90$ & $74.54 \pm 26.60$ & $13972.75 \pm 1214.80$ & $22.56: 1$ \\
\hline
\end{tabular}

Table 12. Physico-chemical properties of the dog excrements mixed with dust rejects in a concentration of $20 \mathrm{~g} . \mathrm{kg}^{-1}$ during anaerobic stabilization (D20) (DM - dry matter, IM - inorganic mater, $\mathrm{OM}$ - organic matter; $\mathrm{NH}_{4}{ }^{+}$- ammonium ions, $\mathrm{N}_{\mathrm{t}}$ - total nitrogen, - - not examined)

\begin{tabular}{cccccccc}
\hline $\begin{array}{c}\text { Storage } \\
\text { (days) }\end{array}$ & $\mathbf{p H}$ & $\begin{array}{c}\mathbf{D M} \\
\mathbf{( \% )}\end{array}$ & $\begin{array}{c}\mathbf{I M} \\
\mathbf{( \% )}\end{array}$ & $\begin{array}{c}\mathbf{O M} \\
\mathbf{( \% )}\end{array}$ & $\begin{array}{c}\mathbf{N H}_{\mathbf{4}}^{+} \\
\text {(mg.kg-1 DM) }\end{array}$ & $\begin{array}{c}\mathbf{N}_{\mathbf{t}} \\
\mathbf{( m g . k g}-\mathbf{D M} \mathbf{~ D M})\end{array}$ & $\mathbf{C : N}$ \\
\hline $\mathbf{0}$ & $9.08 \pm 0.01$ & $35.66 \pm 1.83$ & $11.33 \pm 0.01$ & $88.67 \pm 0,01$ & $219.07 \pm 55.70$ & $40758.43 \pm 1416.02$ & $11.15: 1$ \\
$\mathbf{1}$ & $12.58 \pm 0.04$ & $43.11 \pm 1.51$ & $58.48 \pm 9.02$ & $41.52 \pm 9,02$ & $41.04 \pm 42.79$ & $13041.67 \pm 2498.12$ & $16.22: 1$ \\
$\mathbf{2}$ & $12.68 \pm 0.01$ & $46.81 \pm 0.17$ & $62.89 \pm 2.38$ & $37.11 \pm 2,38$ & 10.19 & $4555.21 \pm 936.46$ & $42.69: 1$ \\
$\mathbf{3}$ & $12.64 \pm 0.01$ & $44.10 \pm 1.95$ & $57.53 \pm 1.39$ & $42.47 \pm 1,39$ & $20.65 \pm 14.40$ & $6868.28 \pm 1649.01$ & $32.67: 1$ \\
$\mathbf{7}$ & $12.36 \pm 0.01$ & $45.50 \pm 0.21$ & $60.84 \pm 0.47$ & $39.16 \pm 0,47$ & $140.34 \pm 9.77$ & $26830.72 \pm 6801.00$ & $7.73: 1$ \\
$\mathbf{8}$ & $10.63 \pm 0.01$ & $45.22 \pm 0.48$ & $62.63 \pm 2.26$ & $37.37 \pm 2,26$ & $131.32 \pm 2.98$ & $14216.80 \pm 5981.61$ & $15.04: 1$ \\
$\mathbf{9}$ & $10.12 \pm 0.01$ & $45.60 \pm 1.23$ & $60.40 \pm 4.67$ & $39.60 \pm 4.67$ & 82.59 & $16161.23 \pm 10202.44$ & $16.18: 1$ \\
$\mathbf{1 0}$ & $10.06 \pm 0.02$ & $46.75 \pm 1.09$ & $63.09 \pm 0.21$ & $36.91 \pm 0.21$ & $85.65 \pm 10.62$ & $13399.86 \pm 759.04$ & $14.14: 1$ \\
$\mathbf{1 4}$ & $9.82 \pm 0.01$ & $52.21 \pm 1.08$ & $65.03 \pm 0.80$ & $34.97 \pm 0.80$ & 14.28 & $19371.98 \pm 1147.30$ & $9.21: 1$ \\
$\mathbf{7 3}$ & $8.97 \pm 0.02$ & $87.17 \pm 0.47$ & $40.50 \pm 1.92$ & $59.50 \pm 1.92$ & $2.20 \pm 0.08$ & $12440.27 \pm 566.93$ & $24.43: 1$ \\
\hline
\end{tabular}

Table 13. Physico-chemical properties of the dog excrements mixed with dust rejects in a concentration of 20 g. $\mathrm{kg}^{-1}$ during anaerobic stabilization (D70) (DM - dry matter, IM - inorganic mater, $\mathrm{OM}$ - organic matter; $\mathrm{NH}_{4}{ }^{+}$- ammonium ions, $\mathrm{N}_{\mathrm{t}}$ - total nitrogen, - - not examined) 


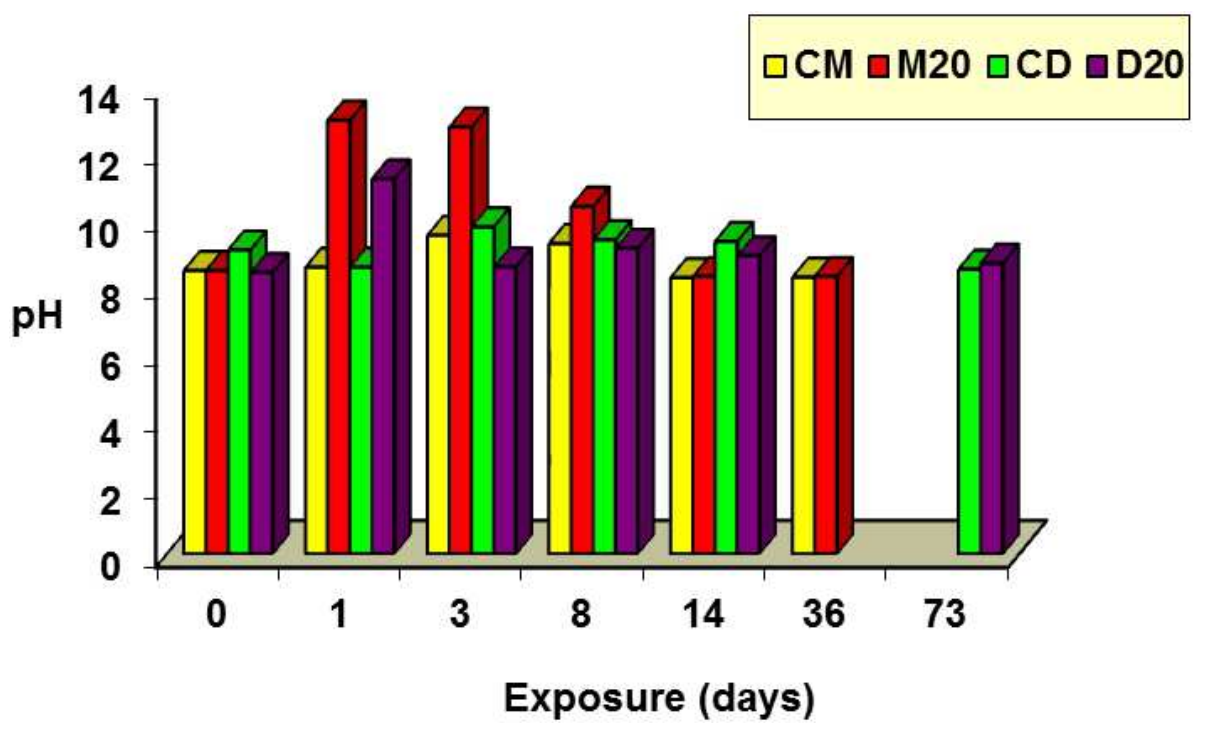

Fig. 1. Comparison of the changes in $\mathrm{pH}$ of organic material during anaerobic stabilisation with or without dust rejects

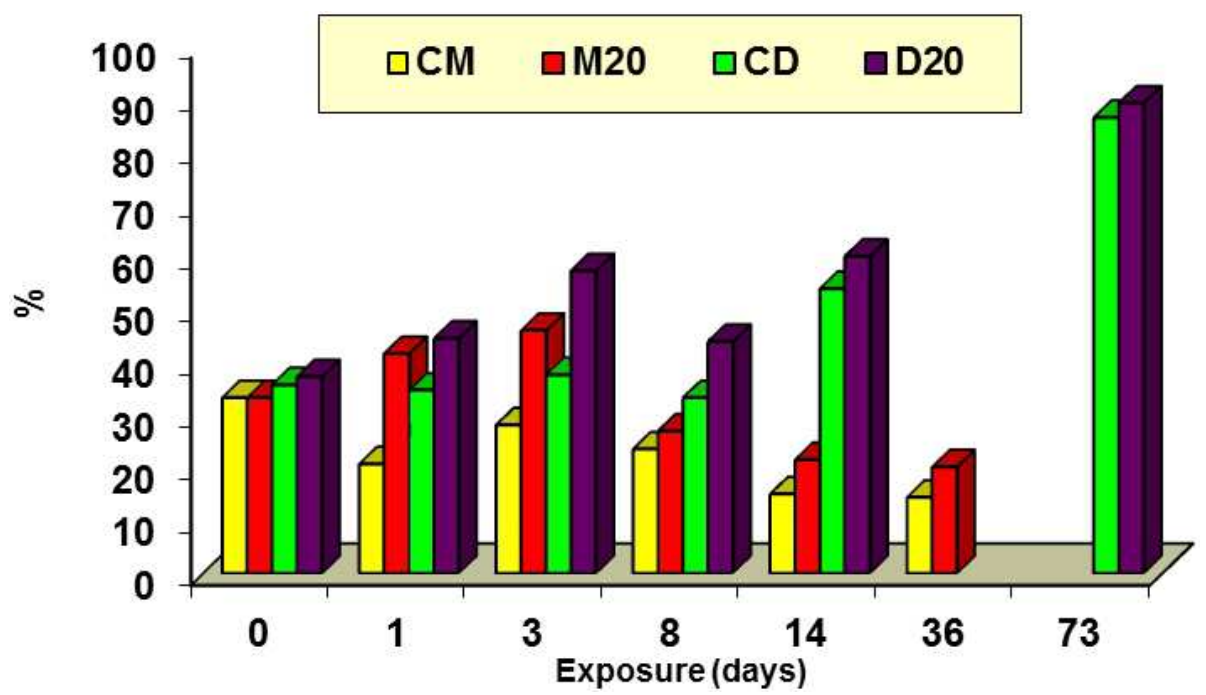

Fig. 2. Comparison of the changes in DM of organic material during anaerobic stabilisation with or without dust rejects 


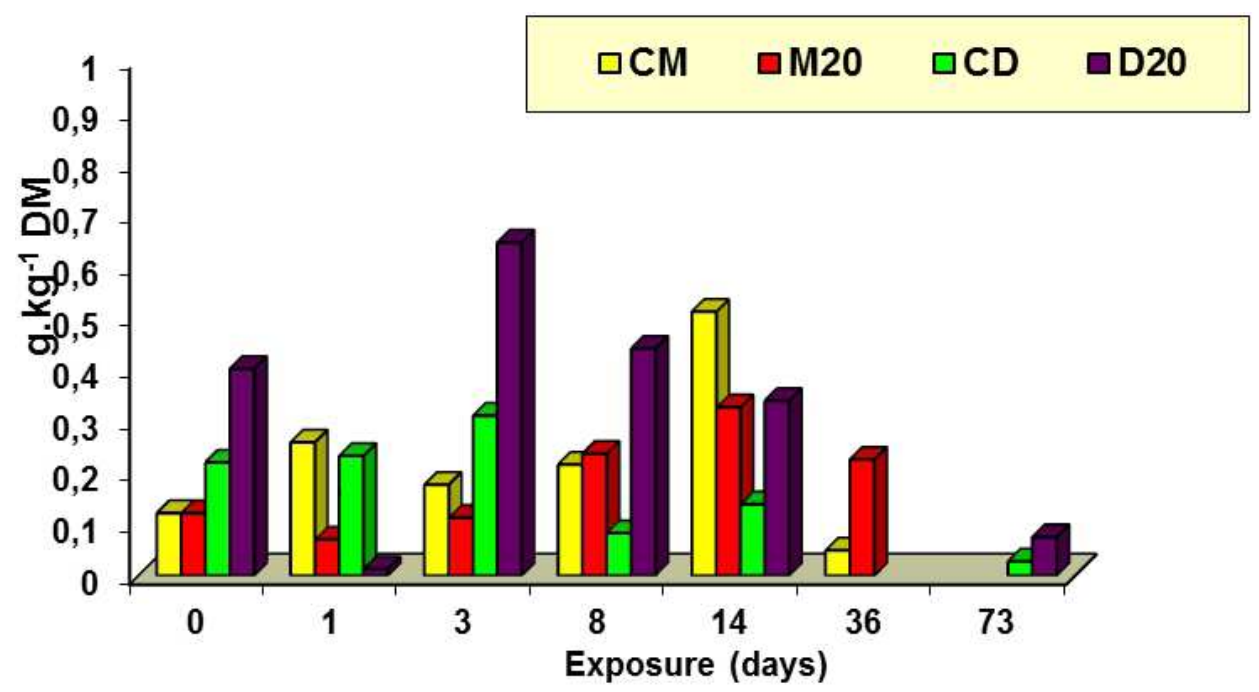

Fig. 3. Comparison of the changes in $\mathrm{NH}_{4}{ }^{+}$of organic material during anaerobic stabilisation with or without dust rejects

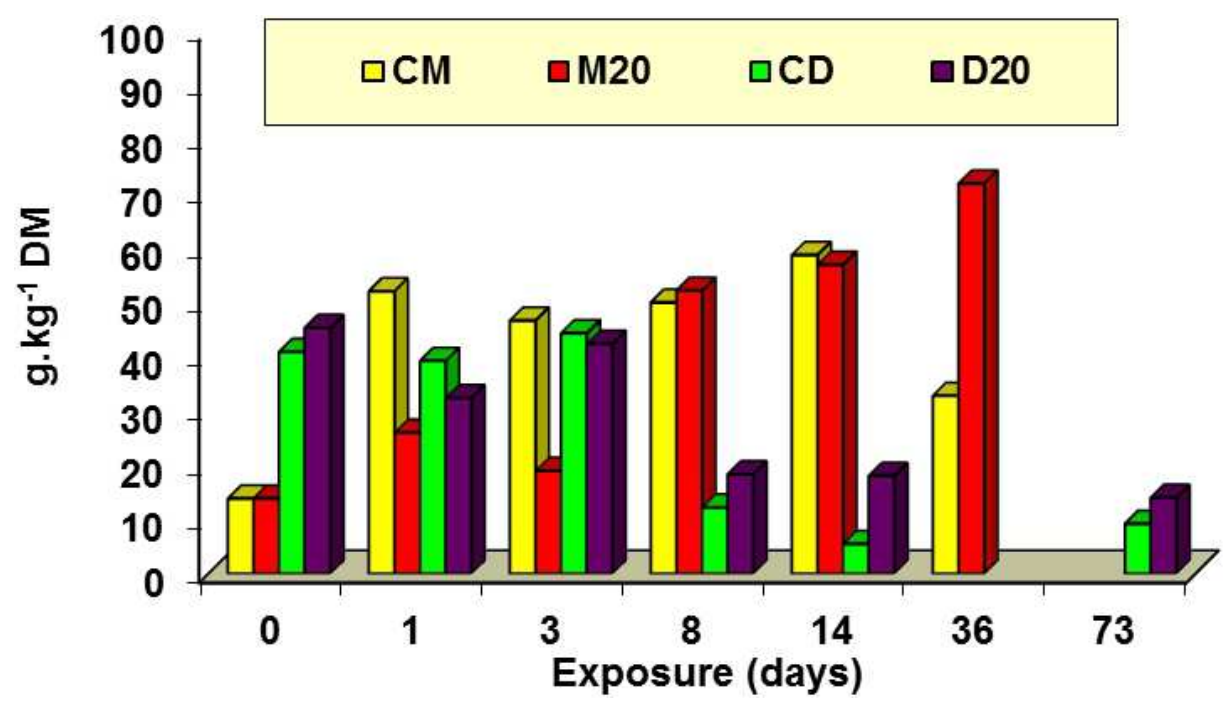

Fig. 4. Comparison of the changes in $\mathrm{N}_{\mathrm{t}}$ of organic material during anaerobic stabilisation with or without dust rejects 


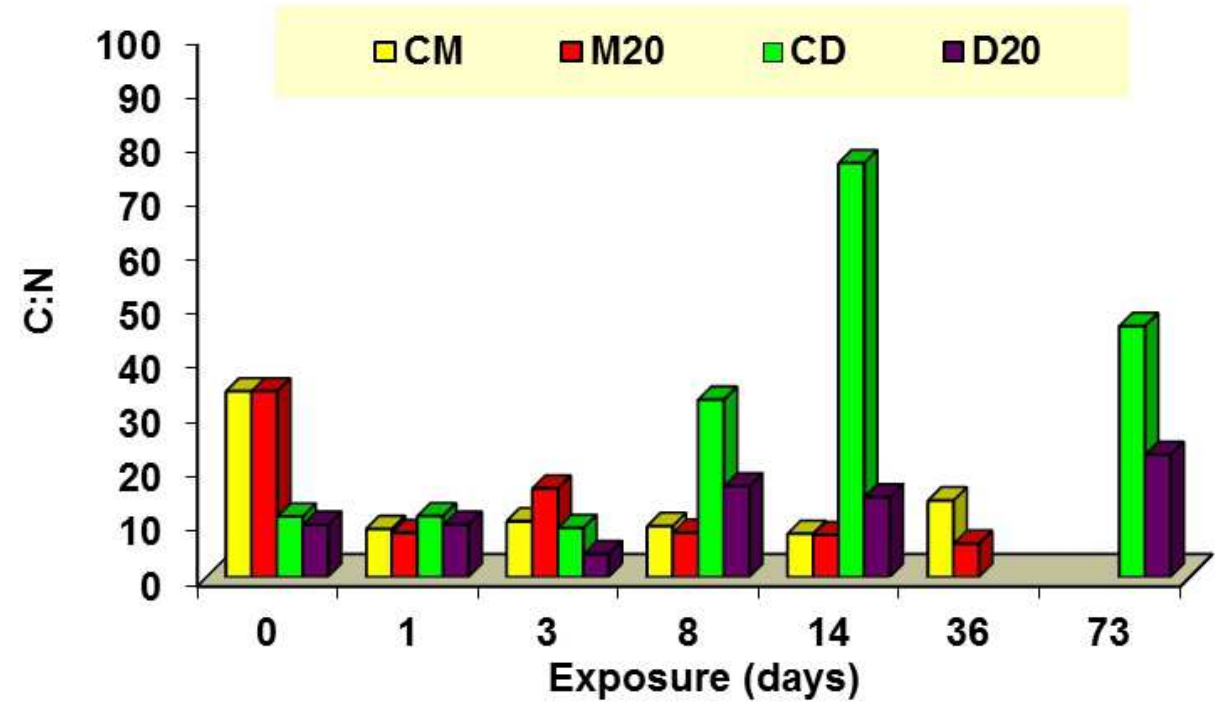

Fig. 5. Comparison of the changes in C:N ratio of organic material during anaerobic stabilisation with or without dust rejects

The Table 14 indicates that a 24 hour after application of both types of lime at concentration 20 g.kg-1 manure more than $80 \%$ of model unembryonated $A$. suum eggs were devitalised. A. suum eggs were totally devitalised as early as till 36 days after application of lime in manure. $58.13 \pm 6.89 \%$ of eggs were devitalised in the control without dust reject in the end of experiment.

\begin{tabular}{cccc}
\hline $\begin{array}{c}\text { Storage } \\
\text { (days)) }\end{array}$ & \multicolumn{3}{c}{ Demaged A. suum eggs (x\% $\mathbf{0}$ SD) } \\
\cline { 2 - 4 } & $\mathbf{C M}$ & ML20 & M20 \\
\hline $\mathbf{0}$ & $16.43 \pm 1.14$ & $16.43 \pm 1.14$ & $16.43 \pm 1.14$ \\
$\mathbf{1}$ & $36.31 \pm 2.46$ & $82.41 \pm 8.49^{* * *}$ & $80.68 \pm 6.75^{* * *}$ \\
$\mathbf{3}$ & $55.10 \pm 10.72^{* *}$ & $87.23 \pm 11.06^{* * *}$ & $89.85 \pm 5.10^{* * *}$ \\
$\mathbf{8}$ & $59.14 \pm 1.74^{* *}$ & $98.96 \pm 1.80^{* * *}$ & $82.22 \pm 16.78^{* * *}$ \\
$\mathbf{1 4}$ & $56.11 \pm 19.64^{*}$ & $97.13 \pm 3.77^{* * *}$ & $97.33 \pm 4.62^{* * *}$ \\
$\mathbf{3 6}$ & $58.13 \pm 6.89^{* *}$ & $100^{* * *}$ & $100^{* * *}$ \\
\hline
\end{tabular}

Table 14. Survival of A. suum eggs during anaerobic stabilisation of the dog excrements with or without lime (* Significance at the level $\mathrm{P}<0.05$; ${ }^{* *}$ Significance at the level $\mathrm{P}<0.01$; *** Significance at the level $\mathrm{P}<0.001$ )

For the sanitation of animal excrenemts, the use of dust rejects from lime production, at more affordable price than quality lime, is very suitable. An application of dust rejects to the mixed dogs' excrements at a concentration of $20 \mathrm{~g} . \mathrm{kg}^{-1}$ of organic wastes, resulted in a 
devitalisation of $65.65 \pm 2.84 \%$ and at a concentration of 70 g.kg-1 $77.05 \pm 2.36 \%$ of model unembryonated $A$. suum eggs within 24 hours (Table 15). A. suum eggs were totally devitalised as early as within 8 days in dogs' excrements after application of dust rejects at a concentration of 70 g. kg-1 and within 21 days after application of dust rejects at a concentration of 20 g. kg-1 due to the changes in physical and chemical properties of the stabilised materials (Tables 12,13). $57.23 \pm 3.21 \%$ of eggs were devitalised in the control without dust reject in the end of experiment (Table 15).

\begin{tabular}{cccc}
\hline \multirow{2}{*}{ Storage (days) } & \multicolumn{3}{c}{ Demaged A. suum eggs $(\mathbf{x} \% \mathbf{0} \mathbf{S D})$} \\
\cline { 2 - 4 } & $\mathrm{CD}$ & $\mathrm{P} 20$ & $\mathrm{P} 70$ \\
\hline $\mathbf{0}$ & $12.62 \pm 1.14$ & $12.62 \pm 1,14$ & $12.62 \pm 1.14$ \\
$\mathbf{1}$ & $35.70 \pm 2.46$ & $65.65 \pm 2.84^{* *}$ & $77.05 \pm 2.36^{* * *}$ \\
$\mathbf{2}$ & $54.43 \pm 10.66^{*}$ & $68.65 \pm 3.89^{* *}$ & $82.30 \pm 4.81^{* * *}$ \\
$\mathbf{3}$ & $67.00 \pm 2.55^{* *}$ & $75.15 \pm 1.21^{* *}$ & $87.60 \pm 3.98^{* * *}$ \\
$\mathbf{7}$ & $62.65 \pm 4.03^{* *}$ & $76.25 \pm 5.41^{* *}$ & $97.13 \pm 3.97^{* * *}$ \\
$\mathbf{8}$ & $59.80 \pm 2.71^{*}$ & $76.93 \pm 2.69^{* * *}$ & $100^{* * *}$ \\
$\mathbf{9}$ & $61.87 \pm 2.90^{*}$ & $82.30 \pm 4.81^{* * *}$ & $100^{* * *}$ \\
$\mathbf{1 0}$ & $62.85 \pm 4.03^{*}$ & $85.69 \pm 1.45^{* * *}$ & $100^{* * *}$ \\
$\mathbf{1 4}$ & $61.96 \pm 3.26^{*}$ & $95.69 \pm 6.35^{* * *}$ & $100^{* * *}$ \\
$\mathbf{2 1}$ & $55.65 \pm 2.36^{*}$ & $100^{* * *}$ & $100^{* * *}$ \\
$\mathbf{7 3}$ & $57.23 \pm 3.21^{*}$ & $100^{* * *}$ & $100^{* * *}$ \\
\hline
\end{tabular}

Tab. 15. Survival of A. suum eggs during anaerobic stabilisation of manure with or without lime ( ${ }^{*}$ Significance at the level $\mathrm{P}<0.05 ;{ }^{* *}$ Significance at the level $\mathrm{P}<0.01$; ${ }^{* *}$ Significance at the level $\mathrm{P}<0.001$ )

Our experiment showed that stabilisation of organic wastes with dust rejects result in complete devitalisation of A. suum eggs (Table 14,15). The most important physico-chemical factors affecting viability of helminth eggs include $\mathrm{pH}$ and ammonia. We observed the highest $\mathrm{pH}$ and ammonia content especially in the organic wastes treated with tested types of lime. One of our previous studies (Ondrašovič et al., 2002) on the effect of ammonium hydroxide on $A$. suum eggs showed that at $10 \%$ concentration of $\mathrm{NH}_{4} \mathrm{OH}, \mathrm{pH} 12.16$ and exposure time $180 \mathrm{~min}$. approx. 94 \% A. suum eggs were devitalised. Pescon and Nelson (2005) also reported that environmentally relevant concentrations of ammonia may significantly increase the rate of Ascaris eggs inactivation during alkaline stabilization.

\section{Conclusion}

Processes of slurry anaerobic stabilization represent an effective method in terms of energy, since the substantial portion of energy present in easily decomposable organic constituents of the substrate is acquired in the form of biogas. Non-decomposed organic matter is well stabilized from hygienic point of view. Anaerobic stabilization increases the proportion of biogenic element (especially nitrogen) converting stabilized excrements into quality fertilizer. Anaerobically stabilized pig slurry stored in lagoon significantly influence the quality and quantity of grasses, depending on the dose of slurry used and on weather conditions. From the nutritional point of view, the sludge (sediment) from ground lagoon is 
also important for plants (Valocká et al., 2000). The high amount of nitrogen is apparently the result of the decomposition process going on in lagoon.

Hazard of contamination of field fertilized with the lagoon effluent increases when raw slurry is used for fertilization of soil or pastures. When slurry is processed in a wastewater treatment plant, parasitic eggs concentrated in solid fraction. It is therefore necessary to pay a proper attention to slurry processing.

The anaerobic stabilization and the use of dust rejects from lime production, at more affordable price than quality lime dust, were demonstrated to be very suitable for the sanitation of organic wastes from animal production and dog excrements. This way of treatment is thus not associated with a risk of dissemination, survival and potential spread of developmental stages of endoparasites to the environment via stabilized organic wastes

\section{Acknowledgements}

We would like to thank CARMEUS Ltd. for providing lime and people who allowed us to gather data needed for elaboration of this chapter. This study was financially supported by the project VEGA No. 2/0147/10.

\section{References}

Antolová, D., Reiterová, K., Miterpáková, M., Stanko, M., Dubinský, P. (2004). Circulation of Toxocara spp. in suburban and rural ecosystems in the Slovak Republic. In Veterinary Parasitology. Vol. 126, no. 3, p. 317-324.

Bremner, J. M. (1996). Nitrogen - total. In D. L. Sparks (Ed.), Methods of Soil Analysis (pp. 1085-1121). Madison, WI: SSSA Inc..

Crompton, D. W. (1999). How much human helminthiasis is there in the world? Journal of Parasitology, 85, 397-403.

Cherepanov, A. A. (1982). Methods of Laboratory Centrals of Cleaning Plants on Farms. Kolos. Moscow (In Russian).

Despommier, D. (2003). Toxocariasis: Clinical aspects, epidemiology, medical ecology and molecular aspects. Clin. Microbiol. Rev. 16, 265-272.

Dubinský, P., Krupicer, I., Levkut, M., Švický, E., Dvorožňáková, E., Revajová, V., Vasilková, Z., Kováč, G., Reiterová, K., Lenhardt, L'., Ondrejka, R., Papajová, I., Moncol, D. J. (2000). Influence of Ascaris suum infection on ruminants. In: Dubinský, P., Juriš, P., Moncol, D. J. (Eds.): Environmental protection against the spread of pathogenic agents of diseases through the wastes of animal production in the Slovak Republic. Harlequine, Ltd., Košice, p. 143-160.

Eckert, J. (1992). Dauerformen von Parasiten als umwelthygienisches Problem, in: Eckert, J., Kutzer, E., Rommel, M., Bürger, H.J., Körting, W. (Eds.), Veterinärmedizinische Parasitologie, 4. Aufl., Verlag Paul Parey, Berlin, 87-107.

Juriš, P., Vasilková, Z., Krupicer, I., Plachý, P., Sasáková, N. (1996). Hygienic and ecological aspects of mesophilic stabilization of swine liquid excrements under the operating conditions. In Hygienic and ecological problems in relation to veterinary Medicine, Košice, Slovak republic, p. 119-128 (In Slovak). 
Juriš, P., Rataj, D., Ondrašovič, M., Sokol, J., Novák, P. (2000). Sanitary and ecological requirements on recycling of organic wastes in agriculture. Vyd. Michala Vaška, Prešov, 1-178 (in Slovak).

Krupicer, I., Valocká, B., Vasilková, Z., Sabová, M., Papajová, I., Dubinský, P. (2000). Contamination and survival of helminth eggs in pig slurry and influence of the lagoon effluent on soil and plant parasitic nematodes. In: Dubinský, P., Juriš, P., Moncol, D. J. (Eds.): Environmental protection against the spread of pathogenic agents of diseases through the wastes of animal production in the Slovak Republic. Harlequine, Ltd., Košice, p. 79-93.

Lauková, A., Juriš, P., Vasilková, Z., Papajová, I. (2000). Treatment of sanitary-important bacteria by bacteriocin substance V24 in cattle dung water. Letters in Applied Microbiology, 30, p. 402-405.

Matsuo, J., Nakashio, S. (2005). Prevalence of fecal contamination in sandpits in public parks in Sapporo City, Japan. In Veterinary Parasitology, Vol. 128, p. 115-119.

Miterpáková, M., Dubinský, P. Reiterová, K., Stanko, M. (2006) Climate and environmental factors influencing Echinococcus multilocularis occurrence in the Slovak Republic. In Annals of Agricultural and Environmental Medicine. Vol. 13, no. 1, p. 235-242.

Mulvaney, R. L. (1996). Nitrogen - inorganic forms. In D. L. Sparks (Ed.), Methods of Soil Analysis (pp. 1123-1184). Madison, WI: SSSA Inc..

Navarro, A. F., Cegarra, J., Roig, A., Garcia, D. (1993). Relationships between organic matter and carbon contents of organic wastes. Bioresource Technology, 44, 203-207.

Ondrašovič, M., Juriš, P., Papajová, I., Ondrašovičová, O., Ďurečko, R., Vargová, M. (2002): Lethal effect of selected disinfectants on Ascaris suum eggs. Helminthologia, 39, pp. 205-209.

Papajová, I., Juriš, P. (2009). The effect of composting on the survival of parasitic germs. In: Pereira, J. C., Bolin, J. L. (Eds.) Composting: Processing, Materials and Approaches. New York : Nova Science Publishers, p. 124-171. ISBN 978-1-60741-438-4.

Pescon, B. M., Nelson, K. L. (2005). Inactivation of Ascaris suum eggs by ammonia. Environ. Sci. Technol., 39, pp. 7909-7914.

Plachý, P., Juriš, P. (1995). Use of polyurethane carrier for assessing the survival of helminth eggs in liquid biological sludges. Vet. Med. 40, 323-326.

Sasáková, N., Juriš, P., Papajová, I., Vargová, M., Ondrašovičová, O., Ondrašovič, M., Kašková, A., Szabová, E. (2005). Parasitological and bacteriological risks to animal and human health arising from waste-water treatment plant. Helminthologia, 42, p. 137-142.

Schwartzbrod, J., Stien, J. L., Bouhoum, K., Baleux, B. (1989). Impact of wastewater treatment on helminth eggs. Water Science and Technology, 21, 295-297. STATISTICA 6.0, StatSoft Inc., USA.

STATISTICA 6.0, StatSoft Inc., USA.

Tofant, A., Vučemilo, M., Hadžiosmanović, M., Križanić, J. (1999). Liquid manure: A surface water pollutant [Einfluß der düngung landwirtschaftlicher flächen mit schweineguille auf die wasserqualitait in naheliegenden gewässern] Tierarztliche Umschau 54, 148-150. 
Valocká, B., Dubinský, P., Papajová, I., Sabová, M. (2000). Effect of anaerobically digested pig slurry from lagoon on soil and plant nematode communities in experimental conditions. Helminthologia, 37, p. 53-57. 


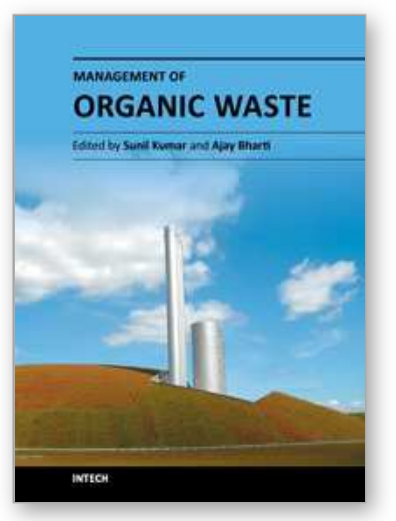

\author{
Management of Organic Waste \\ Edited by Dr. Sunil Kumar
}

ISBN 978-953-307-925-7

Hard cover, 198 pages

Publisher InTech

Published online 01, February, 2012

Published in print edition February, 2012

This book reports research on the utilization of organic waste through composting and vermicomposting, biogas production, recovery of waste materials, and the chemistry involved in the processing of organic waste under various processing aspects. A few chapters on collection systems and disposal of wastes have also been included.

\title{
How to reference
}

In order to correctly reference this scholarly work, feel free to copy and paste the following:

Ingrid Papajová and Peter Juriš (2012). The Sanitation of Animal Waste Using Anaerobic Stabilization, Management of Organic Waste, Dr. Sunil Kumar (Ed.), ISBN: 978-953-307-925-7, InTech, Available from: $\mathrm{http}: / / w w w . i n t e c h o p e n . c o m / b o o k s / m a n a g e m e n t-o f-o r g a n i c-w a s t e / t h e-s a n i t a t i o n-o f-a n i m a l-w a s t e-u s i n g-$ anaerobic-stabilization

\section{INTECH}

open science | open minds

\author{
InTech Europe \\ University Campus STeP Ri \\ Slavka Krautzeka 83/A \\ 51000 Rijeka, Croatia \\ Phone: +385 (51) 770447 \\ Fax: +385 (51) 686166 \\ www.intechopen.com
}

\author{
InTech China \\ Unit 405, Office Block, Hotel Equatorial Shanghai \\ No.65, Yan An Road (West), Shanghai, 200040, China \\ 中国上海市延安西路65号上海国际贵都大饭店办公楼 405 单元 \\ Phone: +86-21-62489820 \\ Fax: $+86-21-62489821$
}


(C) 2012 The Author(s). Licensee IntechOpen. This is an open access article distributed under the terms of the Creative Commons Attribution 3.0 License, which permits unrestricted use, distribution, and reproduction in any medium, provided the original work is properly cited. 